was to investigate safety of belimumab in patients with active SLE in daily clinical practice.

Methods We included patients with diagnosis of SLE (ACR criteria) treated at Medicarte IPS from March 2015 to October 2016. Medicarte is a referral centre for the integral medical care and pharmaco-surveillance of patients under biologic therapies in 13 cities in Colombia. Clinical information was obtained from electronic records. Adverse events (AE) were carefully evaluated during treatment.

Results Thirty three patients (all female) with active SLE were included. Mean age was $38.0 \pm 11.8$ years, and mean disease duration was $10.6 \pm 9.2$ years. Main refractory manifestations were musculoskeletal (100\%), renal (45\%), and mucocutaneous (42\%). Background medications included MMF (87\%), antimalarials (84\%), MTX (72\%), azathioprine (39\%) and RTX (33\%). Mean follow-up under belimumab treatment was 7.9 \pm 5.6 cycles. Mean prednisone doses were $12.0 \pm 11 \mathrm{mg} / \mathrm{d}$. Only $8(24 \%)$ out of 33 patients developed any AE. With a mean exposure time of 5.72 months, $\mathrm{AE}$ incidence rate, expressed as events per $100 \mathrm{p} /$ months was 4.2 (Figure 1). The most common AE were: infusion reactions (3), urinary (2), and respiratory infections (1), herpes zoster (1) and mild pancytopenia (1). None of the patients stopped belimumab due $\mathrm{AE}$

Conclusions Belimumab was safe in clinical practice setting; only a few number of mild side AE were recorded. None of the patients discontinued belimumab treatment due AE.

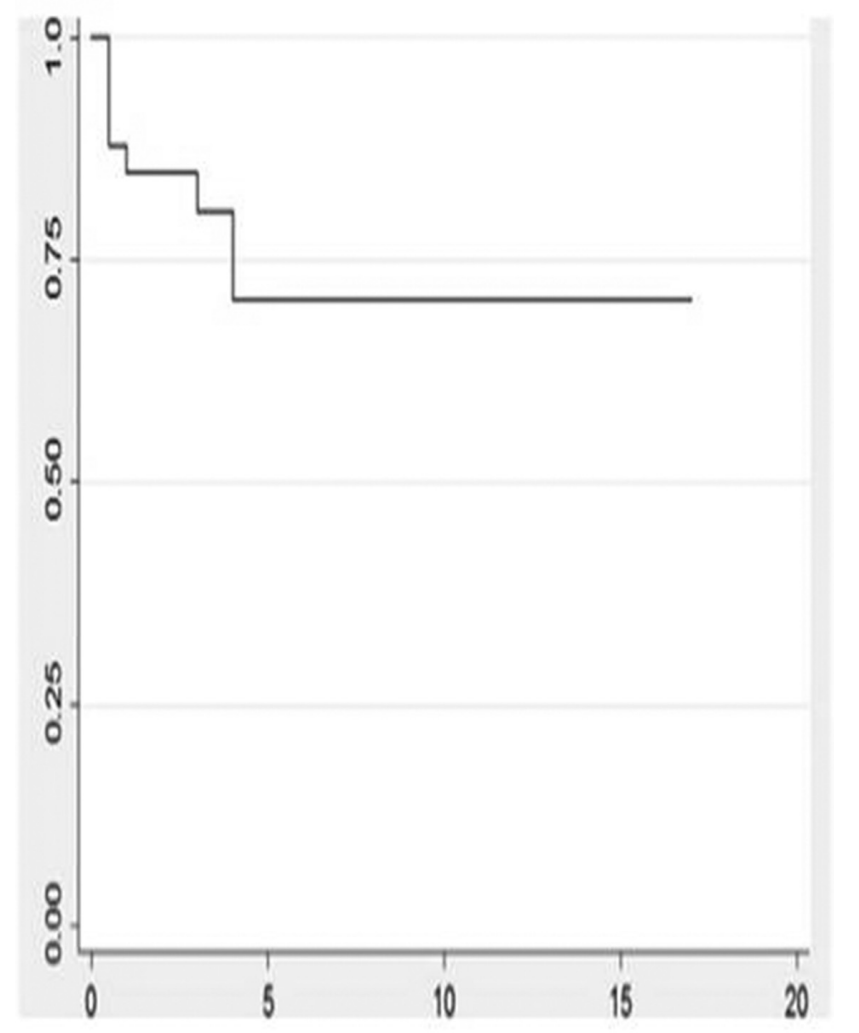

follow:vp(months)

\section{$86 \quad$ MESENCHYMAL STEM CELLS INDUCE LYMPHOCYTES APOPTOSIS INDEPENDENT OF BIM AND BCL-XL IN LUPUS MICE}

${ }^{1} S$ Huang*, ${ }^{2} \mathrm{D}$ Wang, ${ }^{1} \mathrm{~L}$ Sun. ${ }^{1}$ Drum Tower Clinical Medical College of Nanjing Medical University, Department of Immunology and Rheumatology, Nanjing, China; ${ }^{2}$ Affiliated Drum Tower Hospital of Nanjing University Medical School, Department of Immunology and Rheumatology, nanjing, China

\subsection{6/lupus-2017-000215.86}

Background and aims Mesenchymal stem cells (MSCs) have recently been used successfully in humans to control a lot of diseases. However, the mechanisms involved in their immunomodulatory effects remain a matter of debate. Here we explored whether lymphocytes apoptosis involved in the therapeutic effects of UC-MSCs in lupus mice.

Methods $1^{\wedge} 10^{6}$ of human UC-MSCs were injected into B6. lpr mice via tail vein and 6, 24 hours and 4 weeks later, all the mice were sacrificed, the apoptosis of lymphocyte in peripheral blood and spleen tissues as well as the expressions of Bim and Bcl-xl were detected by FACS, the immune cell subpopulations and cytokines in serum were also examined at 6 and 24 hours, respectively. The curative effects were assessed 4 weeks later.

Results UC-MSCs ameliorated disease progression of lupus mice at 4 weeks, increasing the percentage of Treg while downregulating Tfh, plasma cells and Th1 cells, decreasing spleen weight and repairing kidney lesion. UC-MSCs promoted lymphocyte apoptosis in peripheral blood and spleen at 6 and 24 hours, and reduced serum TGF- $\beta 1$ levels, but did not affect Bim and Bcl-xl expressions in CD4+ and CD8 + T cells. Meanwhile, the percentage of Treg was significantly increased in the MSCT group at both 6 and 24 hours. Reductions in the proportions of plasma cells, Th1, Th2 cells were also evident at 24 hours after MSCs infusion.

Conclusions UC-MSCs exhibit extensive pro-apoptosis properties against lymphocytes in B6.lpr mice, which may offer a form of immunomodulatory therapy for lupus.

\section{SELECTIVE AND ORALLY AVAILABLE SMALL MOLECULE INHIBITORS OF TLR7 AND 8 FOR THE TREATMENT OF LUPUS}

${ }^{1} \mathrm{~S}$ Ishizaka*, ${ }^{2} \mathrm{Q}$ Chen, ${ }^{2} \mathrm{D}$ Liu, ${ }^{3}$ L Hawkins, ${ }^{4} \mathrm{~S}$ Fujimoto, ${ }^{4} \mathrm{~J}$ Moriya, ${ }^{4} \mathrm{~A}$ Inoue, ${ }^{4} \mathrm{M}$ Kihara, ${ }^{4} \mathrm{~K}$ Hagiwara. 'Eisai Inc., Target Validation, Andover, USA; ${ }^{2}$ Eisai Inc., Neuroscience Discovery, Andover, USA; ${ }^{3}$ Eisai Inc., Medicinal Chemistry, Andover, USA; ${ }^{4}$ Eisai Tsukuba Research Laboratories, hDAC, Tsukuba, Japan

\subsection{6/lupus-2017-000215.87}

Background and aims The toll-like receptors (TLRs) are critical participants in vertebrate innate immune recognition of pathogen-associated molecular patterns (PAMPs). Diverse ligands act as "danger signals" detected by this component of the innate immune system. TLR7 and 8 are located in the endosomes of specific immune subpopulations, and are activated by singlestranded RNA from viruses or by autologous RNA fragments bound to immune complexes, inducing the generation of cytokines such as interferons (specifically IFN-alpha) and IL-6. Strong genetic evidence supports variants in TLR7 as contributors to development of systemic lupus erythematosus (SLE). Methods In vitro and in vivo assays were used to guide development of potent and specific small molecule inhibitors. 